\title{
Selección y control del factor humano en empresas de construcción civil
}

\begin{abstract}
RESUMEN
Aunque la construcción civil tiene patrones muy singulares, la administración de personal no sigue necesariamente los métodos más adecuados de selección y control de trabajadores/operarios. Esta industria debe contar con especialistas en diversos campos de la construcción tales como en: puentes, carreteras, puertos, vías férreas, etc. El mayor aporte de la presente investigación se basa en la propuesta de selección y control de trabajadores en base a la gestión por competencias, ya que la construcción en el Perú está basada en el conocimiento empírico de su tarea cotidiana. Es así que se propone una estructura del perfil del operario de acuerdo al área en el que debe trabajar, la descripción del cargo, habilidades cognitivas, físicas, nivel educacional y conocimientos generales que deba tener para mejorar la eficiencia y rentabilidad en la ejecución de una obra de construcción civil.
\end{abstract}

Palabras claves: Competencias, perfil del trabajador de construcción civil.

SELECTION AND CONTROL OF HUMAN FACTORS IN CIVIL CONSTRUCTION COMPANIES

\section{ABSTRACT}

Although the construction has very singular landlords, the personnel administration necessarily does not follow the methods most suitable of selection and control of workers/workers. This industry must have specialists in diverse fields of the construction such as in: bridges, highways, ports, railroads, etc. The greater contribution of the present investigation is based on the proposal of selection and control of workers on the basis of the management by competitions, since the construction in Peru is based on the empirical knowledge of its daily task. It is so a structure of the profile of the worker according to the area sets out in which it must work, the description of the position, mental, physical abilities, educational level and general knowledge that must have to improve the efficiency and yield in the execution of a civil construction site.

Keywords: Competitions, profile of the worker of civil construction.

\section{INTRODUCCIÓN}

El desarrollo inmenso que han tenido los sectores de construcción y minería en nuestro país en estos últimos 15 años, concibe la importancia de la Administración de personal y de los recursos financieros en Obras de ingeniería en la ejecución de grandes proyectos.

Si bien son muchas las causas y los factores que contribuyen a que las obras de construcción civil sean deficientes, uno de los más importantes es el relacionado a los recursos humanos con los que cuenta este tipo de proyectos. Es por ello que la selección del personal para trabajos en construcción civil constituye una materia de fundamental importancia en todo el proceso de planeación. Se han empleado mecanismos y técnicas concebidos por la moderna teoría administrativa, pero aun existen deficiencias y concepciones que limitan contar con un adecuado reclutamiento y selección de personal dirigidos a identificar e incorporar a las empresas a quienes cuenten con los conocimientos, habilidades, conductas, experiencias y valores necesarios, cuya integración tendrán repercusión a nivel organizacional.

El problema se centra bajo la siguiente interrogante: ¿Mejorando la forma de selección del factor humano, se logrará incrementar en la planta a trabajadores altamente especializados en los trabajos de ejecución de obras de construcción civil?

\section{Objetivo General}

- Diseñar formatos del perfil requerido para la selección del trabajador de construcción civil en base a las competencias (los conocimientos, habilidades, conductas y experiencias para el puesto).

\section{Objetivos Específicos}

- Identificar la forma con los que se recluta y selecciona actualmente al personal en empresas de construcción civil comprendidas dentro de la sección F, código 45 del CIIU.

- Proponer mapas de puestos, perfiles y competencias, del personal que intervienen en la ejecución de una obra de ingeniería.

1 Doctora Ingeniero Industrial UNMSM, profesora en la facultad de Ingeniería Industrial, Departamento académico de Producción y Gestión Industrial de la UNMSM.

Email: teogaza57@yahoo.es

2 Master en Dirección de Empresas.

Email: ivan tantalean1000@yahoo.com.ar 


\section{Justificación}

El presente trabajo de investigación es importante porque busca desarrollar una propuesta de mejora en el reclutamiento y selección de personal para las empresas de construcción civil, en base al enfoque de competencias laborales, y que influyen en la eficiencia de las empresas constructoras.

\section{MARCO TEÓRICO}

Muchos investigadores, nacionales e internacionales, han realizado un esfuerzo por conceptuar los problemas de la industria de la construcción, estructurando un marco teórico que permita entender mejor qué tipo de producción es la construcción. Esta referencia teórica desarrollada recibe el nombre de "Lean Construction" o "Construcción sin Pérdidas", cuya función es minimizar o eliminar todas aquellas fuentes que implique pérdidas, en el entendido que estas pérdidas implican menor productividad, menor calidad, más costos, etc.

Para Serpell, A \& Alarcon L.F. (2000). "Recientes estudios han demostrado que la planificación representa aproximadamente sólo un $10 \%$ del costo total de un proyecto, sin embargo, regula la ejecución global de éste. Por lo tanto una mala planificación representa la causa principal de los problemas en la construcción, como la no disponibilidad o inadecuada disponibilidad de recursos y, por el contrario, una buena planificación es la clave para lograr una buena eficiencia y efectividad."

Un campo que ha surgido en los últimos 20 años ha sido el de la Gerencia de Construcción Profesional. Dichas firmas, o despachos de profesionales, ofrecen sus servicios desde el diseño hasta la puesta en marcha de proyectos de construcción. Por lo general estas firmas son una variante de las de Ingeniería / Arquitectura o de las de Contratistas Generales, las cuales pueden ser capaces de llevar los roles tanto de ingeniería, arquitectura o de contratista general, pero al servicio del dueño. Aunque la tradición del contratista general sigue prevaleciendo, los Gerentes de Construcción Profesionales son apreciados por algunas empresas ya que sus servicios le pueden proporcionar conocimiento en cada etapa del proyecto, así como absorber parte del riesgo que conlleva el trabajo en sí.

\section{Modelo basado en los Recursos Humanos}

Hoy por hoy las empresas empiezan a aceptar que para sobrevivir y desarrollarse deben revalorar y estimular el desarrollo óptimo de las personas que las integran, en tanto que en ellos reside el recursos estratégico más importante el cual permitirá construir organizaciones no solo más productivas y eficientes, sino organizaciones inteligentes; de aprendizaje y de renovación que harán posible el desarrollo integral de la sociedad. Ya que como plantean Roure y Rodríguez (2000) "son el factor humano de la calidad".

Se hace necesario, entonces, contar con un personal más preparado, que se adapte más rápidamente a la tecnología moderna, que sea proactivo y que realmente sepa interpretar lo que los cambios generan.

La razón principal por la que el recurso humano puede ser entendido como el recurso más preciado y difícilmente remplazable, parte de la simbiosis e interdependencia que existe entre las organizaciones y las personas, Chiavenato (2009) explica: "las organizaciones están conformadas por personas y dependen de ellas para alcanzar sus objetivos y cumplir sus misiones. Para las personas, las organizaciones constituyen el medio para alcanzar varios objetivos personales en el mínimo tiempo y con el menor esfuerzo y conflicto".

El recurso humano establece los objetivos y estrategias empresariales; pero por sobre todo, las personas son la clave para que las empresas, sujetas al cambio continuo, puedan lograr esos objetivos en el mercado competitivo actual.

\section{COMPORTAMIENTO DEL FACTOR HUMANO EN EMPRE- SAS DE CONSTRUCCIÓN}

\section{Administración de personal}

Contrario a lo que mucha gente piensa, la industria de la Construcción, es una industria con grandes déficits de profesionalización, es decir, poca incorporación y utilización de conocimientos científicos o académicos para llevar a cabo su estrategia y gestión. Por lo general (o hasta hace muy poco) no tenían estrategias formalizadas, diseño de procesos, estandarización de procedimientos, sistemas para el control de gestión o la comunicación, y dentro de estos sistemas de gestión de recursos humanos; reclutamiento y selección, inducción, capacitación, evaluación del desempeño, sistema para fijar rentas y compensaciones, descripciones de roles y procesos de coordinación.

"El desarrollo de la actividad de la construcción civil ha determinado una muy particular forma de organizar la actividad de construcción misma, y por ello una forma especial de organizar el trabajo. La peculiaridad de esta actividad radica en aspectos tales como: la necesidad de especialización, capacitación y habilitación, el desplazamiento o la ubicación relativa y el tiempo de duración de los servicios". D. Leg. N0727. La misma norma dice: "Se considera trabajador del régimen de construcción civil a toda 
persona natural que realiza una labor de construcción para otra persona natural o jurídica dedicada a la construcción, con relación de dependencia y a cambio de una remuneración".

\section{Comportamiento del Personal}

La construcción, es una actividad compuesta por un conjunto de procedimientos llevados a cabo para levantar diversos tipos de estructuras. Las principales tendencias actuales en la construcción se alejan del trabajo manual a pie de obra y se orientan hacia el montaje en el lugar de la obra, de componentes mayores y más integrados, fabricados en el lugar de origen.

La construcción en el contexto de la economía es considerada como una actividad estratégica, tanto por su efecto multiplicador en el dinamismo de otros sectores, como por su capacidad de absorción en el corto plazo de mano de obra no calificada.

El sector de la construcción en el Perú, se caracteriza, principalmente, por la presencia de los sindicatos, así para el Ministerio de Trabajo y Promoción del Empleo -MTPE, un sindicato es: "Una organización conformada por un grupo de trabajadores, que crean y aprueban sus propios estatutos, eligen libre y democráticamente a sus representantes y organizan sus actividades, con la finalidad de buscar ventajas para los trabajadores sindicalizados". ¿Cómo se desarrolla la gestión del factor humano en el sector de construcción civil?, ¿La función de un sindicato gremial facilita o no la labor?, ¿Cómo influye la presencia sindical? Se manifiesta que los sindicatos pueden ser defensores del cambio hacia un sistema de alto rendimiento, pero difieren en sus preferencias por ciertas prácticas específicas de recursos humanos.

\section{Gestión por Competencias}

(Becker, 2008) economista norteamericano fue premiado con el Nobel por trabajar con el concepto de Capital humano. Sin embargo por mucho tiempo su trabajo acerca de este tema fue ignorado y criticado por los principales economistas del mundo, quienes no lo consideraban un verdadero analista por dedicar su estudio a dicho concepto al extender el dominio del análisis microeconómico a un amplio rango de comportamiento humano e interacción, incluyendo aquel que no tiene relación con el mercado. Becker comenzó a estudiar las sociedades del conocimiento y concluyó con su estudio señalando, que su mayor tesoro de las empresas era el capital humano que estas poseían, esto es, el conocimiento y las habilidades que forman parte de las personas, su salud y la calidad de sus hábitos de trabajo, además logra definir al capital humano como importante para la productividad de las economías modernas ya que esta productividad se basa en la creación, difusión y utilización del saber.

La gestión por competencias hace la diferencia entre lo que es un curso de capacitación, con una estructura que encierre capacitación, entrenamiento y experiencia que son necesarios de definir para los requerimientos de un puesto o identificar las capacidades de un trabajador o de un profesional.

Figura 1. Gestión por competencias

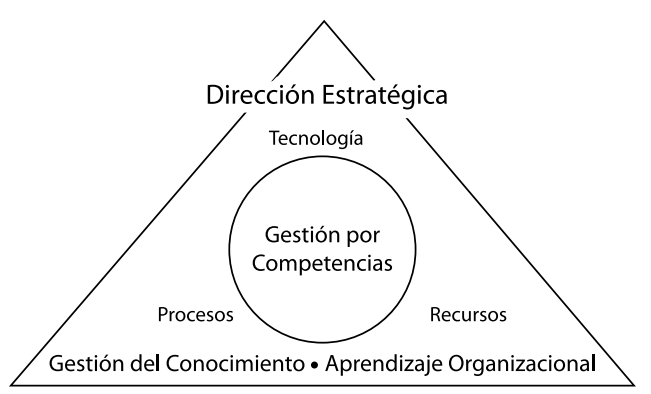

Fuente: Becker, 2008, Capital Humano

El Dr. Mc Clelland, 1998, lideró trabajos sobre pensamiento motivacional, desarrolló las bases de la teoría y de los modelos sobre logros y alcances motivacionales y promocionó mejoras en los métodos de valoración de los empleados.

David McClelland es conocido por describir tres tipos de necesidad motivacional, las cuales él identificó en su libro Human Motivation (1998):

La necesidad de LOGRO (n, LOG). La persona con necesidad de logro se encuentra motivada por llevar a cabo algo difícil, alcanzar algo realmente difícil mediante el reto y desafío de sus propias metas y con ello avanzar en el trabajo. Hay una fuerte necesidad de retroalimentarse de su logro y progreso y una necesidad por sentirse dotado, realizado, gratificado y con talento.

Figura 2. Las motivaciones según McCelland

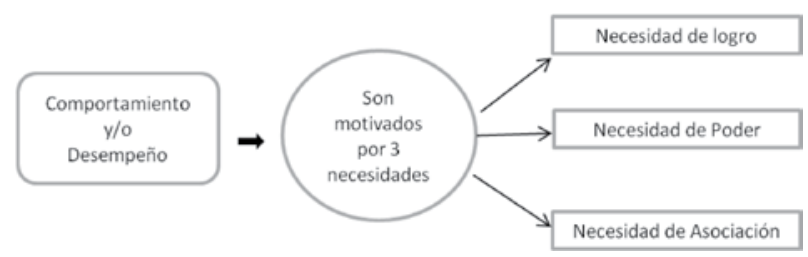

Fuente: Tomado de David C. Mc Celland 1998

Por otra parte, (Chiavenato, 2009), nos detalla los tipos de selección de personal para empresas de construcción civil, según diferentes modelos:

Modelo de colocación: Caracterizada por la existencia de un solo candidato y una sola vacante. El modelo no incluye la alternativa de rechazarlo. El candidato que se presente es admitido, sin sufrir rechazo. 
Modelo de selección: Caracterizada por la existencia de varios candidatos y una sola vacante a ocupar. En este caso cada candidato es comparado con los requisitos que exige el puesto y sólo se presenta dos alternativas: la aprobación o el rechazo. Si el candidato es reprobado se lo elimina del proceso ya que existen otros candidatos para el puesto vacante.

Modelo de clasificación: Se caracteriza por la existencia de varios candidatos para varios puestos de trabajo. Cada candidato es comparado con los requisitos que exige el puesto. Se presentan dos alternativas: ser aprobado o ser rechazado. Si es rechazado, se lo compara con los requisitos de otros puestos que se pretende cubrir, hasta que se agoten los puestos vacantes. En este modelo la organización no lo considera al candidato con interese en un solo puesto, sino como una candidato para la organización y se le podrá colocar en el puesto más adecuado dadas sus características personales.

Modelo del Valor agregado: Este modelo es el que se propone, y va más allá de la simple comparación con el puesto que será ocupado y se enfoca en el abastecimiento y la provisión de competencias a la organización. Cada candidato es visto desde un punto de vista de las competencias individuales que ofrece para elevar las competencias del proyecto. La idea básica es incrementar el portafolio de competencias de las obras de construcción, de modo que se garantice la competitividad de la empresa mediante la culminación efectiva de las obras.

Figura 3. Modelos de selección de personal

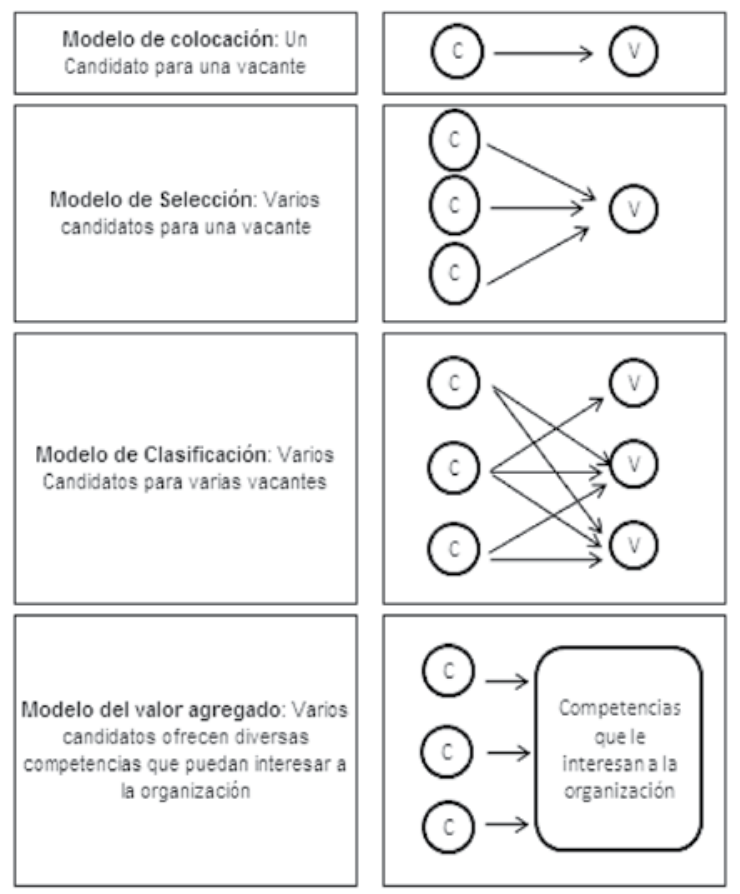

Fuente: Tomado de Chiavenato, 2009

\section{METODOLOGÍA DE LA INVESTIGACIÓN}

Se aplicó el tipo de investigación Descriptiva Explicativa; el propósito fue describir situaciones actuales de comportamiento del personal en las empresas de construcción civil, describiendo las labores que realizan, cuyos eventos se repiten en diferentes formas de desempeño o por mala selección de personal.

Tamayo y Tamayo (2005), define la investigación descriptiva como aquella que: "Comprende la descripción, registros, análisis e interpretación de la naturaleza actual y la composición o proceso de los fenómenos". El enfoque se hace sobre conclusiones dominantes o sobre como una persona, grupo o cosa se conduce o funciona en el presente. "La investigación descriptiva trabaja sobre realidades de hecho y su característica fundamental es la de presentar una definición correcta"

En opinión de Dankhe (1996) los estudios descriptivos buscan especificar las propiedades importantes de personas, grupos, comunidades o cualquier otro fenómeno que sea sometido a análisis. Desde el punto de vista científico, describir es medir. Esto es, en el estudio descriptivo aplicado a los trabajadores de construcción civil se buscó seleccionar una serie de factores que afecten el desarrollo de sus actividades y esta a su vez en el logro de los objetivos de construcción, se buscó determinar:

\section{La metodología de selección de personal}

2. Competencias laborales aplicadas al personal

El método explicativo, nos permitió descartar y explorar las variables que intervienen en el fenómeno que se propone a investigar. Además de describir el fenómeno se tratará de buscar la explicación del comportamiento de las variables. Su metodología es básicamente cualitativa, y su fin último es el descubrimiento de las causas. Como su nombre lo indica, su interés se centra en explicar porque ocurre un fenómeno y en que condiciones se manifiesta, o porque se relacionan dos o mas variables. La aplicación del método explicativo para la presente investigación trató de responder a las siguientes preguntas:

1. ¿Qué variables intervienen en el desempeño del personal obrero, operario, técnico y profesional, para que los trabajos que se les asignan, permita un mal desempeño?

2. ¿Qué efectos se tendrían, si se aplicara un perfil por competencias, en el proceso de selección de personal? 


\section{DISEÑO DE LA INVESTIGACIÓN}

El presente trabajo de investigación busca determinar la importancia de contar con una metodología de selección de personal por competencias aplicado a los trabajadores sector de la construcción civil, así para Marelli, (2000), "Las competencias son capacidades laborales necesarias para realizar un trabajo eficazmente, es decir para producir los resultados deseados para la organización. Está conformada por conocimientos, habilidades, destrezas y comportamientos que los trabajadores deben demostrar para que la organización alcance sus metas y objetivos."

La presente investigación busca proponer las competencias mínimas requeridas para los trabajadores de construcción civil, es que se apoyó en el método inductivo y se aplicó estudios cualitativos, ya que ésta: "se preocupa por el contexto de los acontecimientos, y centran su indagación en aquellos contextos en los que los seres humanos se involucran e interesan, evalúan y experimentan directamente". Vera Vélez, Lamberto (2003).

Con esta metodología se consiguió un acercamiento con los trabajadores de producción en la ejecución de las obras, quienes son los directamente implicados y ven el mundo de la construcción desde su perspectiva; esto constituye su principal atractivo. Se buscó encontrar la relación:

\section{TRABAJADOR - COMPETENCIAS - TRABAJO}

Los estudios cualitativos se caracterizan por : a) la riqueza de información que brinda al investigador, debido a la diversidad de informantes que incluye en el proceso de recopilación de datos y, sobre todo, b) por la profundidad de las preguntas.

Para efectos de la investigación se tuvo que trasladar a las obras de construcción de la empresa, materia de estudio, y en contacto directo con el ambiente de trabajo se identificaron determinados rasgos que caracterizan la labor del trabajador tomando como base un esquema conceptual predeterminado y propósitos ya establecidos en relación al cronograma de avance de obra, se observó el desempeño individual de los trabajadores, se tomó nota de los hechos observados y se comparó con la labor de otros trabajadores y se analizó los resultados con la teoría de gestión por competencias.

En la ejecución del proyecto en obras de construcción civil, las actividades se realizan por frentes de trabajo, que vienen hacer un grupo de trabajadores que desarrollan una actividad en particular del proyecto, ejemplo:
"El frente de trabajo de vigas y encofrados; compuesta por 6 operarios: 3 fierreros y 3 encofradores, desarrollan labores transformación y doblado de fierro corrugados, fierro de temperatura, para posteriormente, el armado de vigas y columnas mediante el uso de madera y clavos.

Figura 2. Frentes de trabajo

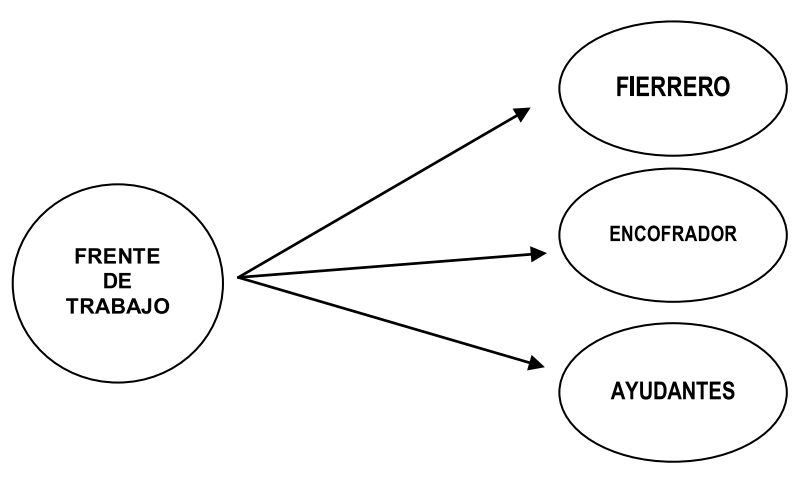

DE LO GENERAL

A LO PARTICULAR

Fuente: Elaboración propia

\section{UNIDAD DE ANÁLISIS}

Para la presente investigación, la unidad de análisis estuvo representada por los trabajadores de construcción civil, que estuvo compuesta por: operarios, técnicos, y profesionales, de una empresa del mismo sector. Las razones para no mencionar el nombre de la empresa, materia de estudio, es con la finalidad de evitar que se ejerza el derecho de propiedad sobre la investigación y los resultados que se obtengan.

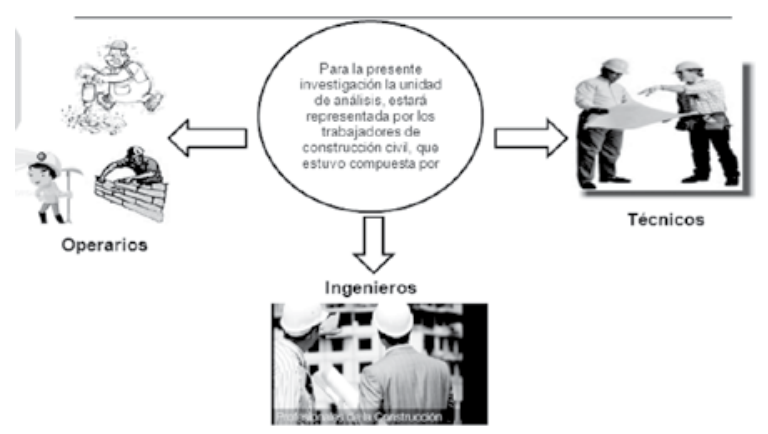

Fuente: Elaboración propia

\section{RESULTADOS}

DISEÑO DEL MODELO PARA LA SELECCIÓN DE PERSONAL POR COMPETENCIAS MOTIVO DE LA INVESTIGACIÓN

El modelo que aplican las empresas constructoras a la fecha son muy simples y generalistas, se concentran sólo en requerimientos de carácter académico, administrativo y legal, mientras que el modelo 
propuesto a través de formatos, permiten identificar además de los requisitos antes mencionados las competencias necesarias para la ocupación de los cargos de acuerdo a sus especialidades.

El enfoque de solución ante la problemática planteada, consiste fundamentalmente en lo siguiente:

1. Aplicación de los perfiles por competencia, en la etapa de la selección, a todo el personal que interviene en la ejecución de un proyecto de construcción civil.

2. Incorporar a la organización o proyecto solo a quienes cuenten con los conocimientos, habilidades, conductas, experiencias y valores necesarios, exigidos por el proyecto en especial.
3. El líder del proyecto, profesional que asumirá la responsabilidad de ejecutar la obra, debe de contar como requisito para su contratación el de poseer conocimiento y experiencia en la gestión por competencias, ya que la empresa contratista tiene como organización la independencia y autonomía de sus obras, debe de integrar la gestión por competencias como parte su política integral de calidad.

A continuación se presenta un ejemplo de los muchos perfiles de competencias requeridos para los puestos en las actividades de la construcción civil y que se encuentran en la investigación realizada y que obra en la biblioteca de la Facultad de Ingeniería Industrial, (ver formatos utilizados).

DISEÑO DEL FORMATO DEL PERFIL PARA EL TRABAJADOR DE CONSTRUCCIÓN CIVIL:

\begin{tabular}{|c|c|}
\hline \multicolumn{2}{|c|}{ Título del Puesto } \\
\hline \multicolumn{2}{|c|}{ ALBAÑIL } \\
\hline \multicolumn{2}{|c|}{ Departamento de Ubicación } \\
\hline \multicolumn{2}{|c|}{ Jefe de Producción / Operaciones } \\
\hline Dependencia & Supervisión \\
\hline Directa & Directa Funcional \\
\hline MAESTRO DE OBRAS & Ayudante de albañilería \\
\hline Indirecta & Indirecta \\
\hline Jefe de Frente / Jefe de Producción & $\mathrm{N} / \mathrm{A}$ \\
\hline \multicolumn{2}{|c|}{ Misión del Puesto } \\
\hline \multicolumn{2}{|c|}{$\begin{array}{l}\text { Organiza y ejecuta la preparación de mezclas, colocación de fierros, vigas, columnas, techos y acabados en condiciones } \\
\text { de seguridad y calidad establecidas }\end{array}$} \\
\hline \multicolumn{2}{|c|}{ Roles más importantes del Puesto } \\
\hline \multicolumn{2}{|l|}{$\begin{array}{l}\text { 1. } \text { Organizar los trabajos de albañilería. } \\
\text { 2. Ejecutar la preparación de mezcla para el leva } \\
\text { 3. } \\
\text { 4. Ejecutar el levantamiento de muros y el vaciad } \\
\text { 5. }\end{array}$} \\
\hline \multicolumn{2}{|l|}{ Relaciones Internas del Puesto } \\
\hline
\end{tabular}




\begin{tabular}{|c|c|c|c|c|c|}
\hline \multicolumn{6}{|c|}{ Relaciones Externas del Puesto } \\
\hline \multicolumn{6}{|c|}{ Zonas de Trabajo } \\
\hline \multicolumn{6}{|c|}{ - Según donde se ejecute la Obra.( Fuera de Lima ) } \\
\hline \multicolumn{6}{|c|}{ Uso de equipos } \\
\hline \multicolumn{6}{|c|}{ Wincher, Mezcladora, Radio. } \\
\hline \multicolumn{6}{|c|}{ PUESTO DE ALBAÑIL } \\
\hline \multicolumn{6}{|c|}{$\begin{array}{l}\text { GRADO REQUERIDO DE COMPETENCIAS } \\
\text { (Esenciales en todos los puestos) }\end{array}$} \\
\hline \multirow{2}{*}{ No. } & \multirow{2}{*}{ Competencia } & & \multicolumn{3}{|c|}{ Grados } \\
\hline & & & Base & Medio & Alto \\
\hline 1 & Orientación al Servicio y al Cliente & & $\mathbf{x}$ & & \\
\hline 2 & Orientación a Resultados & & & & $\mathbf{x}$ \\
\hline 3 & Compromiso (Involucramiento) & & & & $\mathbf{x}$ \\
\hline 4 & Mejora Innovativa & & & & $\mathbf{x}$ \\
\hline 5 & Comportamiento centrado en Valores & & & & $\mathbf{x}$ \\
\hline 6 & Cooperación y Trabajo en Equipo & & & & $\mathbf{x}$ \\
\hline \multicolumn{6}{|c|}{ GRADO REQUERIDO DE COMPETENCIAS COMPUESTAS } \\
\hline \multirow{2}{*}{ No. } & \multirow{2}{*}{ Competencia } & \multicolumn{4}{|c|}{ Grados } \\
\hline & & $\begin{array}{c}\text { No } \\
\text { Requerida } \\
\end{array}$ & Base & Medio & Alto \\
\hline 7 & De manipulación (Cinético corporales) & & & & $\mathbf{x}$ \\
\hline 8 & De Relaciones interpersonales & & & $\mathbf{x}$ & \\
\hline 9 & De Comunicación & & & & $\mathbf{x}$ \\
\hline 10 & Afectivas & & & $\mathbf{x}$ & \\
\hline 11 & De Previsión, Proyección y Planeación & & & $\mathbf{x}$ & \\
\hline 12 & De Organización & & & $\mathbf{x}$ & \\
\hline 13 & De Liderazgo & & $\mathbf{x}$ & & \\
\hline 14 & De Negociación & & $\mathbf{x}$ & & \\
\hline 15 & De Delegación (Empowerment) & $x$ & & & \\
\hline 16 & De creación de redes de relaciones & $x$ & & & \\
\hline 17 & De Emprendimiento & & $x$ & & \\
\hline
\end{tabular}




\begin{tabular}{|c|c|c|c|c|c|}
\hline \multicolumn{6}{|c|}{$\begin{array}{c}\text { RECURSOS CLAVES DE LAS COMPETENCIAS } \\
\text { (Características para la selección de personal y la evaluación del desempeño) }\end{array}$} \\
\hline \multirow[b]{2}{*}{ No. } & \multirow[b]{2}{*}{ Cognitivos y Habilidades } & \multicolumn{4}{|c|}{ Grados de desarrollo } \\
\hline & & Mínimo & Normal & $\begin{array}{r}\text { Medio } \\
\text { Alto } \\
\end{array}$ & Alto \\
\hline 1 & Pensamiento guiado por modelos & & & & $\mathbf{x}$ \\
\hline 2 & Pensamiento analógico & & & & $\mathbf{x}$ \\
\hline 3 & Pensamiento abstracto & & & & $\mathbf{x}$ \\
\hline 4 & Pensamiento creativo & & & $\mathbf{x}$ & \\
\hline 5 & Atención y retención de detalles & & & & $\mathbf{x}$ \\
\hline \multirow[t]{2}{*}{6} & Concentración y Vigilia & & & & $\mathbf{x}$ \\
\hline & Otras habilidades singulares: & & & & \\
\hline \multirow[b]{2}{*}{ No. } & \multirow[b]{2}{*}{ Actitudes, Intereses y Valores } & \multicolumn{4}{|c|}{ Grados de desarrollo } \\
\hline & & Mínimo & Normal & \begin{tabular}{|r|} 
Medio \\
Alto \\
\end{tabular} & Alto \\
\hline 7 & Iniciativa y pro actividad & & & & $\mathbf{x}$ \\
\hline 8 & Tenacidad, perseverancia & & & $\mathbf{x}$ & \\
\hline 9 & Precisión, exactitud, interés en la calidad & & & & $\mathbf{x}$ \\
\hline 10 & Deseo de ayuda & & & & $\mathbf{x}$ \\
\hline 11 & Curiosidad & & $\mathbf{x}$ & & \\
\hline 12 & Aspiraciones, ambición & & & $\mathbf{x}$ & \\
\hline 13 & Honestidad, integridad & & & & $\mathbf{x}$ \\
\hline 14 & Equidad & & & $\mathbf{x}$ & \\
\hline \multirow[b]{2}{*}{ No. } & \multirow[b]{2}{*}{ Afectivos y Psicofísicos } & \multicolumn{4}{|c|}{ Grados de desarrollo } \\
\hline & & Mínimo & Normal & \begin{tabular}{|r|} 
Medio \\
Alto \\
\end{tabular} & Alto \\
\hline 15 & Empatía & & & $\mathbf{x}$ & \\
\hline 16 & Ajuste emocional y adaptabilidad social & & & $\mathbf{x}$ & \\
\hline 17 & Tolerancia a la frustración & & & & $\mathbf{x}$ \\
\hline 18 & Tolerancia a la presión & & & & $\mathbf{x}$ \\
\hline 19 & Tolerancia a la incertidumbre & & & & $\mathbf{x}$ \\
\hline \multirow{2}{*}{ No. } & \multirow{2}{*}{\multicolumn{2}{|c|}{ Recurso Físicos y de Salud Clave }} & \multicolumn{3}{|c|}{ Grados de desarrollo } \\
\hline & & & Normal & $\begin{array}{l}\text { Medio } \\
\text { alto }\end{array}$ & Alto \\
\hline 20 & Resistencia al esfuerzo físico intenso & & & & $\mathbf{x}$ \\
\hline 21 & Fuerza del tronco (Columna, brazos y piernas) & & & & $\mathbf{x}$ \\
\hline 22 & Desarrollo de los sentidos & & & & $\mathbf{x}$ \\
\hline
\end{tabular}




\section{HERRAMIENTAS DE SELECCIÓN Y EVALUACIÓN LISTA DE CHEQUEO \\ PUESTO: ALBAÑIL}

\begin{tabular}{|c|c|c|}
\hline $\begin{array}{l}\text { Herramientas de } \\
\text { Evaluación }\end{array}$ & Responsable & Contenido \\
\hline Formulario de Empleo & RR. HH. & - Información solicitada, con carácter de declaración jurada \\
\hline \multirow{8}{*}{$\begin{array}{l}\text { Certificaciones } \\
\text { Relevantes }\end{array}$} & \multirow{4}{*}{ Externas } & - Educación: Certificados Oficiales. \\
\hline & & - Laboral: De Trabajo y Prácticas \\
\hline & & $\begin{array}{l}\text { Perfil Personal: De testimonios relevantes (anterior empleador, } \\
\text { profesores, instituciones de ayuda, líderes). }\end{array}$ \\
\hline & & $\begin{array}{l}\text { - Seguridad: De Antecedentes Policiales, Judiciales y Penales. } \\
\text { Informe de Seguridad. }\end{array}$ \\
\hline & \multirow{4}{*}{$\begin{array}{c}\text { Avance o } \\
\text { reingreso) } \\
\text { RR. HH. }\end{array}$} & - Asistencia. Puntualidad \\
\hline & & - Amonestaciones. Suspensiones. \\
\hline & & - Reconocimientos y Méritos recibidos. \\
\hline & & - Cursos internos realizados \\
\hline \multirow[b]{2}{*}{ Exámenes de Suficiencia } & Médico & - $\quad$ Examen médico. \\
\hline & Psicológico & $\begin{array}{l}\text { - Perfil de Inteligencia, Habilidades y Destrezas } \\
\text { - } \quad \text { Perfil Psicológico (Rasgos psicológicos, afectivos y sociales } \\
\text { Valores) }\end{array}$ \\
\hline $\begin{array}{l}\text { Formulario Evaluación de } \\
\text { Desempeño }\end{array}$ & Gerente Técnico & $\begin{array}{l}\text { - Evaluación de Desempeño con un Enfoque de Competencias - } \\
\text { Personal de Jefatura y Supervisión }\end{array}$ \\
\hline
\end{tabular}

\section{CONCLUSIONES}

1. Se diseñó perfiles basados en una nueva forma de selección donde cuenten las habilidades, competencias, conductas, experiencias y valores, cuya integración tendrán repercusión a nivel organizacional que serán necesarios para cada especialidad, en el presente resumen so coloca un solo formato como ejemplo, que es el de la albañilería.

2. Esta nueva forma de seleccionar al personal de construcción civil permitirá contar con un adecuado reclutamiento dirigido a identificar e incorporar a las empresas a quienes cuenten con los conocimientos, habilidades para cada puesto y de esta forma evitar la rotación y costos de selección, de tal manera que garanticen el éxito en la realización de sus tareas.

\section{RECOMENDACIONES}

3. Es recomendable la aplicación de las técnicas de selección propuestas, ya que estas permitirán conocer realmente las características de los puestos ofertados de las diferentes obras para cada aspirante a ello y de esta forma tener personal competente y capaz en cada tarea asignada.

4. En todas las obras de construcción civil es recomendable que tengan las empresas constructoras en su base de datos información de los perfiles de los puestos de trabajo que requiera el tipo de obra, para que a través del uso de sistemas de cómputo faciliten tanto la formulación, manejo, mantenimiento y revisión de los perfiles de puesto. 


\section{REFERENCIAS BIBLIOGRÁFICAS}

[1] Becker, Gary Stanley (2008). El capital Humano. USA: University of Chicago Press.

[2] Chiavenato, Idalberto (2009). Gestión del Talento Humano. México D.F: Interamericana Editores S.A. Mc Graw Hill.

[3] Marelli, Anne, (2000). Introducción al análisis y desarrollo de modelos de competencias. Cinterfor-OIT.

[4] McClellan, (1998), Teoría Tres Necesidades De McClelland, visitado en dic. 2009, http:// www.mitecnologico.com/Main
[5] Rodríguez, M y Roure, J. (2000). Aprendiendo de los mejores. Barcelona: Ed. Gestión.

[6] Serpell, A \& Alarcón, L.F (2000). Planificación y Control de Proyectos. Santiago: Ediciones Universidad Católica de Chile.

[7] Tamayo Tamayo, Mario (2005). El Proceso de la Investigación Científica. México. D.F: Limusa.

[8] Vera Vélez, Lamberto. (2003). La Investigación Cualitativa. Disponible en web: http:// ponce.inter.edu/cai/reserva/lvera/INVESTIGACION_CUALITATIVA.pdf. Enero 2011. 